\title{
Conservation laws in anisotropic elasticity I. Basic framework $\dagger$
}

\author{
B Y C.-S. YEH ${ }^{1}$, Y.-C. SH U ${ }^{2}$ A D K.-C. W U ${ }^{3}$ \\ ${ }^{1}$ Department of Civil Engineering and Institute of Applied Mechanics, \\ ${ }^{2}$ Department of Civil Engineering and ${ }^{3}$ Institute of Applied Mechanics, \\ National Taiwan University, Taipei 106, Taiwan, R.O.C.
}

A complete class of first order conservation laws for two dimensional deformations in general anisotropic elastic materials is derived. The derivations are based on Stroh's formalism for anisotropic elasticity. The general procedure proposed by P. J. Olver for the construction of conservation integrals is followed. It is shown that the conservation laws are intimately connected with Cauchy's theorem for complex analytic functions. Real-form conservation laws that are valid for degenerate or non-degenerate materials are given.

\section{Introduction}

It is a well-accepted fact that conservation laws play an important role in mathematics, physics and engineering science from both a theoretical and practical standpoint. Determination of conservation laws of a system of differential equations provides a basic tool in the analysis of the properties of the solutions and associated valuable physical implications. In particular, conservation laws provide a powerful means in elasticity for the study of inhomogeneities including cracks, dislocations, and inclusions within a body. Eshelby (1956) has first introduced the concept of the force on the inhomogeneity of the body by evaluating a path-independent integral representing a conservation law over any surface enclosing the inhomogeneity. This integral, which was also derived independently and named as the $J$ integral by Rice (1968), has been used extensively in the study of crack propagation and proved to be of great practical utility due to its path-independency.

It is never a trivial task, in general, to find out non-trivial conservation laws of a given system of differential equations. However, for systems derivable by variational principles, Noether (1918) has provided a remarkable result that for each infinitesimal transformation which leaves the variational action integral invariant there exists a non-trivial conservation law. Thus, construction of conservation laws can be developed from a direct study of the variational action integral. This is indeed a very powerful tool to derive conservation laws systematically for most physical systems are admitted by certain variational principles. For example, in the case of isotropic hyperelasticity, the strain energy density plays the

$\dagger$ This paper was produced from the authors' disk by using the $\mathrm{T}_{\mathrm{E}} \mathrm{X}$ typesetting system.

Proc. R. Soc. Lond. A (1993) 443, 139-151

(C) 1993 The Royal Society 
role of the lagrangian density in its variational formulation. Thus, homogeneity and isotropy of physical space lead to invariance of the associated action integrals under translation and rotation of physical space respectively. Applying the Noether's theorem and symmetry group theory, Olver (1984a) gave general methods and conditions of the existence of variational and generalized symmetries in elasticity. For linear isotropic elasticity in both two and three dimensions, the general structure of the conservation laws was completely determined by Olver $(1984 b)$. In a subsequent paper, Olver (1988) further extended the result to planar anisotropic elasticity involving only two in-plane displacement components. Because of the restrictions of transformations on the material coordinates and displacements, it appears difficult to generalize Olver's derivation to the more general two dimensional deformations for materials without elastic symmetry.

The aim of this paper is to derive general first-order conservation laws in general two-dimensional anisotropic elastostatics. The deformations considered here depend only on two independent variables but three displacement components are allowed to coexist. The approach by Olver is adopted here but the derivation is carried out using Stroh's formalism (Stroh 1958) for anisotropic elasticity. Both the non-degenerate and degenerate cases such as isotropic elasticity are discussed. The present derivation has the advantage that the conservation integrals obtained can be readily expressed in terms of physical quantities using the orthogonality relations in the Stroh's formalism. In addition, the present derivation can be extended to include thermoelasticity. The extension and applications of the conservation laws derived in this work to thermoelastic crack problems are reported in the following paper.

The plan of this paper is as follows. In $\S 2$ Stroh's formalism is introduced. The general conservation laws are derived in $\S 3$. Some real-form conservation integrals in terms of physical quantities are given in $\S 4$. Finally some concluding remarks are made.

\section{Stroh's formalism}

A brief derivation of Stroh's formalism (Stroh 1958, 1962; Barnett \& Lothe 1973; Chadwick \& Smith 1977; Ting 1986) is introduced in this section for Navier's equations which are independent of the coordinate $x_{3}$ in a fixed cartesian coordinate system $\left(x_{1}, x_{2}, x_{3}\right)$. Hereafter, summation over repeated indices from 1 to 3 is implied unless noted otherwise and a comma stands for partial differentiation; boldfaced symbols represent vectors, tensors or matrices.

Consider the following system of equations

$$
C_{i j k l} u_{k, l j}=0,
$$

where $C_{i j k l}$ is the elasticity tensor. For such equations, the general solution can be expressed as follows (Stroh 1958):

$$
u_{k}=a_{k} f(z), \quad z=x_{1}+p x_{2},
$$

where $p$ and $\boldsymbol{a}$ are constants and $f$ is an arbitrary function of $z$. Substitution of (2.2) into the (2.1) yields

$$
\left(\boldsymbol{Q}+p\left(\boldsymbol{R}+\boldsymbol{R}^{\mathrm{T}}\right)+p^{2} \boldsymbol{T}\right) \boldsymbol{a}=0
$$

Proc. R. Soc. Lond. A (1993) 
in which the superscript $\mathrm{T}$ stands for the transpose of a matrix and

$$
Q_{i k}=C_{i 1 k 1}, \quad R_{i k}=C_{i 1 k 2}, \quad T_{i k}=C_{i 2 k 2} .
$$

For non-trivial solutions of $\boldsymbol{a}$, it follows that

$$
\operatorname{det}\left(\boldsymbol{Q}+p\left(\boldsymbol{R}+\boldsymbol{R}^{\mathrm{T}}\right)+p^{2} \boldsymbol{T}\right)=0,
$$

where det is the determinant of a matrix. Equation (2.5) provides six eigenvalues $p$ and eigenvectors $\boldsymbol{a}$. These eigenvalues and eigenvectors appear as three complex conjugate pairs for stable elastic materials for which the elasticity tensor is positive definitive. For definiteness, $p_{k}(k=1-3)$ will be assumed to have positive imaginary parts. The materials for which Stroh's eigenvalues are distinct are called non-degenerate in this paper. The materials with Stroh's eigenvalues appearing as a double or triple root of (2.3) are called degenerate. Isotropic material is an example of the degenerate materials. The discussions to be followed hereafter will be for non-degenerate materials except at the end of $\S 3$ where degenerate materials will be treated.

Introduce the vector $\boldsymbol{b}_{k}$ for $k=1-3$ as

$$
\left.\boldsymbol{b}_{k}=\left(\boldsymbol{R}^{\mathrm{T}}+p_{k} \boldsymbol{T}\right) \boldsymbol{a}_{k}=-p^{-1}\left(\boldsymbol{Q}+p_{k} \boldsymbol{R}\right) \boldsymbol{a}_{k} \quad \text { (no sum over } k\right),
$$

and define the $3 \times 3$ matrices $\boldsymbol{A}$ and $\boldsymbol{B}$ by

$$
\begin{aligned}
& \boldsymbol{A}=\left(\boldsymbol{a}_{1}, \boldsymbol{a}_{2}, \boldsymbol{a}_{3}\right), \\
& \boldsymbol{B}=\left(\boldsymbol{b}_{1}, \boldsymbol{b}_{2}, \boldsymbol{b}_{3}\right) .
\end{aligned}
$$

Based on the assumption that the eigenvalues $p$ are distinct, the general solution for the system (2.1) can be expressed as

$$
u_{i}=\sum_{k=1}^{3} A_{i k} f_{k}\left(z_{k}\right)+\sum_{k=1}^{3} \bar{A}_{i k} \overline{f_{k}\left(z_{k}\right)}, \quad i=1-3,
$$

in which $f_{k}$ are arbitrary analytic functions in their complex arguments $z_{k}=$ $x_{1}+p_{k} x_{2}$; the overbar denotes complex conjugate and $\boldsymbol{A}$ is given in (2.7). The matrix $\boldsymbol{B}$ is associated with the stress functions $\psi_{i}$ as

$$
\psi_{i}=\sum_{k=1}^{3} B_{i k} f_{k}\left(z_{k}\right)+\sum_{k=1}^{3} \bar{B}_{i k} \overline{f_{k}\left(z_{k}\right)}, \quad i=1-3,
$$

The stress functions are related to the stresses $\sigma_{i j}$ by

$$
\begin{aligned}
& \sigma_{i 1}=-\psi_{i, 2}, \\
& \sigma_{i 2}=\psi_{i, 1} .
\end{aligned}
$$

The matrices $\boldsymbol{A}$ and $\boldsymbol{B}$ can be shown to have the following relations (Stroh 1962):

$$
\begin{aligned}
& \boldsymbol{A}^{\mathrm{T}} \boldsymbol{B}+\boldsymbol{B}^{\mathrm{T}} \boldsymbol{A}=\boldsymbol{I}=\overline{\boldsymbol{A}}^{\mathrm{T}} \overline{\boldsymbol{B}}+\overline{\boldsymbol{B}}^{\mathrm{T}} \overline{\boldsymbol{A}}, \\
& \boldsymbol{A}^{\mathrm{T}} \overline{\boldsymbol{B}}+\boldsymbol{B}^{\mathrm{T}} \overline{\boldsymbol{A}}=0=\overline{\boldsymbol{A}}^{\mathrm{T}} \boldsymbol{B}+\overline{\boldsymbol{B}}^{\mathrm{T}} \boldsymbol{A}, \\
& \boldsymbol{A} \boldsymbol{B}^{\mathrm{T}}+\overline{\boldsymbol{A}} \overline{\boldsymbol{B}}^{\mathrm{T}}=\boldsymbol{I}=\boldsymbol{B} \boldsymbol{A}^{\mathrm{T}}+\overline{\boldsymbol{B}} \overline{\boldsymbol{A}}^{\mathrm{T}}, \\
& \boldsymbol{A} \boldsymbol{A}^{\mathrm{T}}+\overline{\boldsymbol{A}} \overline{\boldsymbol{A}}^{\mathrm{T}}=0=\boldsymbol{B} \boldsymbol{B}^{\mathrm{T}}+\overline{\boldsymbol{B}} \overline{\boldsymbol{B}}^{\mathrm{T}} .
\end{aligned}
$$

Proc. R. Soc. Lond. A (1993) 
By (2.15), one can let

$$
\begin{aligned}
& \boldsymbol{A} \boldsymbol{A}^{\mathrm{T}}=-\frac{1}{2} \mathrm{i} \boldsymbol{H}, \\
& \boldsymbol{B} \boldsymbol{B}^{\mathrm{T}}=\frac{1}{2} \mathrm{i} \boldsymbol{L},
\end{aligned}
$$

where $\boldsymbol{H}$ and $\boldsymbol{L}$ are real symmetric positive-definite matrices. Equation (2.15) implies

$$
\boldsymbol{A} B^{\mathrm{T}}=\frac{1}{2}(\boldsymbol{I}-\mathrm{i} \boldsymbol{S})
$$

where $\boldsymbol{S}$ is real. The real matrices $\boldsymbol{H}, \boldsymbol{L}$ and $\boldsymbol{S}$ can be calculated directly from the elastic constants (Barnett \& Lothe 1973).

By applying (2.13) and (2.14) to (2.9) and (2.10), one has

$$
f_{i}=A_{k i} \psi_{k}+B_{k i} u_{k}
$$

In addition, differentiating (2.20) with respect to $x_{1}$ and $x_{2}$ yields

$$
\begin{aligned}
\varphi_{i}\left(z_{i}\right) & =f_{i}^{\prime}\left(z_{i}\right) \\
& =A_{k i} \sigma_{k 2}+B_{k i} u_{k, 1} \\
& =p_{i}^{-1}\left(-A_{k i} \sigma_{k 1}+B_{k i} u_{k, 2}\right) \quad(\text { no sum over } i) .
\end{aligned}
$$

In the sequel (2.22) will be utilized to express the conservation laws in terms of such physical quantities as displacements and stresses.

\section{Conservation laws}

Olver (1986) has shown that the non-trivial first-order conservation laws can be expressed as

$$
\nabla \cdot \boldsymbol{T}=T_{1,1}+T_{2,2}=\sum_{i=1}^{3} Q_{i} E_{i}=0
$$

where

$$
\boldsymbol{T}(\boldsymbol{x}, \boldsymbol{u}, \nabla \boldsymbol{u})=\left(T_{1}, T_{2}\right), \quad E_{i}=C_{i j k l} u_{k, l j}
$$

Equation (3.1) holds identically for certain non-zero coefficients $Q_{i}$ which are functions of $\boldsymbol{x}, \boldsymbol{u}$, and $\nabla \boldsymbol{u}$. In (3.1) $\boldsymbol{T}$ is a first-order conserved density with components $T_{1}$ and $T_{2}$. Moreover, according to Olver (1984b), the components of a trivial conservation law of the planar system (2.1) can be written as

$$
T_{1}=R_{, 2}, \quad T_{2}=-R_{, 1},
$$

where $\mathrm{R}(\boldsymbol{x}, \boldsymbol{u})$ is an arbitrary smooth function of $\boldsymbol{x}$ and $\boldsymbol{u}$.

In the following we shall derive the first-order conservation laws satisfying (3.1) using Stroh's formalism. The general procedure proposed by Olver $(1984 a)$ will be followed. The procedure is briefly outlined here. Expand (3.1) by the chain rule as

$$
\left.\frac{\partial T_{k}}{\partial x_{k}}\right|_{\boldsymbol{u}, \nabla \boldsymbol{u}}+\left.\frac{\partial T_{k}}{\partial u_{i}}\right|_{\boldsymbol{x}, \nabla \boldsymbol{u}} u_{i, k}+\left.\frac{\partial T_{k}}{\partial u_{i, j}}\right|_{\boldsymbol{x}, \boldsymbol{u}} u_{i, j k}=\sum_{i=1}^{3} Q_{i} E_{i} .
$$

Since the first two terms of the left-hand side of (3.4) do not contain second Proc. R. Soc. Lond. A (1993) 
derivatives of $\boldsymbol{u}$, we must have

$$
\begin{gathered}
\left.\frac{\partial T_{k}}{\partial x_{k}}\right|_{\boldsymbol{u}, \nabla \boldsymbol{u}}+\left.\frac{\partial T_{k}}{\partial u_{i}}\right|_{\boldsymbol{x}, \nabla \boldsymbol{u}} u_{i, k}=0, \\
\left.\frac{\partial T_{k}}{\partial u_{i, j}}\right|_{\boldsymbol{x}, \boldsymbol{u}} u_{i, j k}=\sum_{i=1}^{3} Q_{i} E_{i}=0 .
\end{gathered}
$$

The conserved density $\boldsymbol{T}$ can thus be found by first solving (3.6) to determine its dependence on $\nabla \boldsymbol{u}$. Equation (3.5) can then be used to further establish the additional dependence of $\boldsymbol{T}$ on $\boldsymbol{x}$ and $\boldsymbol{u}$.

To solve (3.6), let us write $\varphi_{k}$ in (2.21) as

$$
\varphi_{k}=\varphi_{k 1}+\mathrm{i} \varphi_{k 2} \text {, }
$$

where $\varphi_{k 1}$ and $\varphi_{k 2}$ are real. According to (2.22) and (2.23), we can change variables from $u_{i, 1}$ and $u_{i, 2}$ to $\varphi_{i 1}$ and $\varphi_{i 2}$. Effecting the change of variables in (3.1) and expanding the resulting equation leads to

$$
T_{1, \varphi_{k 1}} \varphi_{k 1,1}+T_{1, \varphi_{k 2}} \varphi_{k 2,1}+T_{2, \varphi_{k 1}} \varphi_{k 1,2}+T_{2, \varphi_{k 2}} \varphi_{k 2,2}=0
$$

where

$$
T_{1, \varphi_{k i}}=\frac{\partial T_{1}}{\partial \varphi_{k i}}, \quad T_{2, \varphi_{k i}}=\frac{\partial T_{2}}{\partial \varphi_{k i}}, \quad k=1-3, \quad i=1,2 .
$$

Since, for each fixed $k, \varphi_{k}$ is an analytic function in $z_{k}$, the following relations hold:

$$
\begin{aligned}
\varphi_{k 1,2} & =p_{k 1} \varphi_{k 1,1}-p_{k 2} \varphi_{k 2,1} \quad(\text { no sum over } k), \\
\varphi_{k 2,2} & =p_{k 2} \varphi_{k 1,1}+p_{k 1} \varphi_{k 2,1} \quad(\text { no sum over } k), \\
p_{k} & =p_{k 1}+\mathrm{i} p_{k 2},
\end{aligned}
$$

where $p_{k 1}$ and $p_{k 2}$ are the real and imaginary parts of $p_{k}$ respectively. Substitution of (3.10)-(3.12) into (3.8) yields

$$
\left(T_{1, \varphi_{k 1}}+p_{k 1} T_{2, \varphi_{k 1}}+p_{k 2} T_{2, \varphi_{k 2}}\right) \varphi_{k 1,1}+\left(T_{1, \varphi_{k 2}}+p_{k 1} T_{2, \varphi_{k 2}}-p_{k 2} T_{2, \varphi_{k 1}}\right) \varphi_{k 2,1}=0 .
$$

Since (3.13) holds as an identity in $\varphi_{k 1,1}$ and $\varphi_{k 2,1}$, we must have

$$
\left.\begin{array}{ll}
T_{1, \varphi_{k 1}}+p_{k 1} T_{2, \varphi_{k 1}}+p_{k 2} T_{2, \varphi_{k 2}}=0 & (\text { no sum over } k), \\
T_{1, \varphi_{k 2}}+p_{k 1} T_{2, \varphi_{k 2}}-p_{k 2} T_{2, \varphi_{k 1}}=0 & \text { (no sum over } k) .
\end{array}\right\}
$$

Further analysis shows that all mixed partial derivatives of $T_{1}$ and $T_{2}$ with respect to $\varphi_{k i}$ and $\varphi_{l j}$ vanish for $k \neq l$. Indeed, differentiating (3.14) with respect to $\varphi_{l 1}$ and $\varphi_{l 2}$, we have

$$
\left.\begin{array}{l}
T_{1, \varphi_{k 1} \varphi_{l 1}}=-p_{k 1} T_{2, \varphi_{k 1} \varphi_{l 1}}-p_{k 2} T_{2, \varphi_{k 2} \varphi_{l 1}}, \\
T_{1, \varphi_{k 1} \varphi_{l 2}}=-p_{k 1} T_{2, \varphi_{k 1} \varphi_{l 2}}-p_{k 2} T_{2, \varphi_{k 2} \varphi_{l 2}}, \\
T_{1, \varphi_{k 2} \varphi_{l 1}}=-p_{k 1} T_{2, \varphi_{k 2} \varphi_{l 1}}+p_{k 2} T_{2, \varphi_{k 1} \varphi_{l 1}}, \\
T_{1, \varphi_{k 2} \varphi_{l 2}}=-p_{k 1} T_{2, \varphi_{k 2} \varphi_{l 2}}+p_{k 2} T_{2, \varphi_{k 1} \varphi_{l 2}} .
\end{array}\right\}
$$

The fact that the above equations also hold as we interchange $k$ and $l$ leads to

$$
\boldsymbol{P q}=\mathbf{0},
$$

Proc. R. Soc. Lond. A (1993) 
where

$$
\boldsymbol{P}=\left[\begin{array}{cccc}
p_{l 1}-p_{k 1} & p_{l 2} & -p_{k 2} & 0 \\
-p_{l 2} & p_{l 1}-p_{k 1} & 0 & -p_{k 2} \\
p_{k 2} & 0 & p_{l 1}-p_{k 1} & p_{l 2} \\
0 & p_{k 2} & -p_{l 2} & p_{l 1}-p_{k 1}
\end{array}\right], \quad \boldsymbol{q}=\left\{\begin{array}{l}
T_{2, \varphi_{k 1} \varphi_{l 1}} \\
T_{2, \varphi_{k 1} \varphi_{l 2}} \\
T_{2, \varphi_{k 2} \varphi_{l 1}} \\
T_{2, \varphi_{k 2} \varphi_{l 2}}
\end{array}\right\}
$$

Moreover, the determinant of $\boldsymbol{P}$ can be shown to be

$$
\operatorname{det} \boldsymbol{P}=\left(p_{l 1}-p_{k 1}\right)^{4}+2\left(p_{l 1}-p_{k 1}\right)^{2}\left(p_{l 2}^{2}+p_{k 2}^{2}\right)+\left(p_{k 2}^{2}-p_{l 2}^{2}\right)^{2}>0
$$

(no sum over $k, l$ ) if $p_{k} \neq p_{l}$ and $k \neq l$. Hence, we must have $T_{2, \varphi_{k i} \varphi_{l j}}=0$ for $k \neq l$ and $i, j=1,2$. Furthermore, from (3.15), the mixed partial derivatives of $T_{1}$ with respect to $\varphi_{k i}$ and $\varphi_{l j}$ also vanish for $k \neq l$. Therefore, $T_{1}$ and $T_{2}$ can be expressed as

$$
T_{1}=\sum_{k=1}^{3} T_{1}^{(k)}\left(\varphi_{k 1}, \varphi_{k 2}, \boldsymbol{x}, \boldsymbol{u}\right), \quad T_{2}=\sum_{k=1}^{3} T_{2}^{(k)}\left(\varphi_{k 1}, \varphi_{k 2}, \boldsymbol{x}, \boldsymbol{u}\right),
$$

where $T_{j}^{(k)}$ are arbitrary functions. With (3.16), (3.14) is simply a pair of CauchyRiemann equations for the complex function

$$
F_{k}=\left(T_{1}^{(k)}+p_{k 1} T_{2}^{(k)}\right)+\mathrm{i}\left(-p_{k 2} T_{2}^{(k)}\right) \quad(\text { no sum over } k),
$$

and (3.16) can be expressed as

$$
\begin{aligned}
& T_{1}=\sum_{k=1}^{3}\left(F_{k 1}+\frac{p_{k 1}}{p_{k 2}} F_{k 2}\right), \\
& T_{2}=\sum_{k=1}^{3} \frac{-1}{p_{k 2}} F_{k 2}, \\
& F_{k}=F_{k 1}+\mathrm{i} F_{k 2}, \quad k=1-3,
\end{aligned}
$$

where $F_{k 1}$ and $F_{k 2}$ are the real and imaginary parts of the function $F_{k}=$ $F_{k}\left(\varphi_{k}, \boldsymbol{x}, \boldsymbol{u}\right)$.

To further determine the dependence of $F_{k}$ on $\boldsymbol{x}$ and $\boldsymbol{u}$, let us define

$$
\begin{aligned}
\tilde{z}_{k} & =x_{1}-p_{k} x_{2}, \\
\partial_{\tilde{z}_{k}} & =\frac{1}{2}\left(\partial_{x_{1}}-p_{k}^{-1} \partial_{x_{2}}\right),
\end{aligned}
$$

so that (3.5) can be expressed as

$$
\frac{1}{2} \operatorname{Re}\left\{\sum_{k, j} \frac{\mathrm{i} p_{k}}{p_{k 2}}\left(2 \partial_{\tilde{z}_{k}} F_{k}+2 F_{k_{, u_{j}}} \partial_{\tilde{z}_{k}} u_{j}\right)\right\}=0,
$$

where Re denotes the real part. Equation (3.23) can be further rewritten as

$$
\operatorname{Re}\left\{\sum_{k, j, r} \frac{\mathrm{i} p_{k}}{p_{k 2}}\left[2 \partial_{\tilde{z}_{k}} F_{k}+\left(A_{j r}\left(1-p_{r} / p_{k}\right) \varphi_{r}+\bar{A}_{j r}\left(1-\bar{p}_{r} / p_{k}\right) \bar{\varphi}_{r}\right) F_{k, u_{j}}\right]\right\}=0 .
$$


Since, for each fixed $k$, there are no such terms as $\varphi_{k} \mho\left(\varphi_{k}\right)$, where $\mho\left(\varphi_{k}\right)$ is an arbitrary analytic function of its argument $\varphi_{k}$. Based on this fact, the subsequent analysis will be extremely simplified by using the following lemma (cf. Olver 1988).

Lemma 1. Suppose $\varphi_{1}, \varphi_{2}$ and $\varphi_{3}$ are independent complex variables and the functions $V_{i}, H_{i j}$ and $G_{i j}$ are complex analytic functions with the associated single complex variable for $i, j=1-3$, respectively, then

$$
\begin{aligned}
& \operatorname{Re}\left\{V_{1}\left(\varphi_{1}\right)+\bar{\varphi}_{1} H_{11}\left(\varphi_{1}\right)+\varphi_{2} G_{12}\left(\varphi_{1}\right)+\bar{\varphi}_{2} H_{12}\left(\varphi_{1}\right)+\varphi_{3} G_{13}\left(\varphi_{1}\right)\right. \\
& +\bar{\varphi}_{3} H_{13}\left(\varphi_{1}\right)+V_{2}\left(\varphi_{2}\right)+\varphi_{1} G_{21}\left(\varphi_{2}\right)+\bar{\varphi}_{1} H_{21}\left(\varphi_{2}\right)+\bar{\varphi}_{2} H_{22}\left(\varphi_{2}\right) \\
& +\varphi_{3} G_{23}\left(\varphi_{2}\right)+\bar{\varphi}_{3} H_{23}\left(\varphi_{2}\right)+V_{3}\left(\varphi_{3}\right)+\varphi_{1} G_{31}\left(\varphi_{3}\right)+\bar{\varphi}_{1} H_{31}\left(\varphi_{3}\right) \\
& \left.+\varphi_{2} G_{32}\left(\varphi_{3}\right)+\bar{\varphi}_{2} H_{32}\left(\varphi_{3}\right)+\bar{\varphi}_{3} H_{33}\left(\varphi_{3}\right)\right\}=0,
\end{aligned}
$$

if and only if

$$
\begin{aligned}
& H_{11}\left(\varphi_{1}\right)=\mathrm{i} h_{11} \varphi_{1}+s_{11}, \quad H_{22}\left(\varphi_{2}\right)=\mathrm{i} h_{22} \varphi_{2}+s_{22}, \quad H_{33}\left(\varphi_{3}\right)=\mathrm{i} h_{33} \varphi_{3}+s_{33}, \\
& G_{12}\left(\varphi_{1}\right)=g_{12} \varphi_{1}+r_{12}, \quad G_{21}\left(\varphi_{2}\right)=-g_{12} \varphi_{2}+r_{21}, \quad G_{31}\left(\varphi_{3}\right)=-g_{13} \varphi_{3}+r_{31}, \\
& H_{12}\left(\varphi_{1}\right)=h_{12} \varphi_{1}+s_{12}, \quad H_{21}\left(\varphi_{2}\right)=-\bar{h}_{12} \varphi_{2}+s_{21}, \quad H_{31}\left(\varphi_{3}\right)=-\bar{h}_{13} \varphi_{3}+s_{31}, \\
& G_{13}\left(\varphi_{1}\right)=g_{13} \varphi_{1}+r_{13}, \quad G_{23}\left(\varphi_{2}\right)=g_{23} \varphi_{2}+r_{23}, \quad G_{32}\left(\varphi_{3}\right)=-g_{23} \varphi_{3}+r_{32}, \\
& H_{13}\left(\varphi_{1}\right)=h_{13} \varphi_{1}+s_{13}, \quad H_{23}\left(\varphi_{2}\right)=h_{23} \varphi_{2}+s_{23}, \quad H_{32}\left(\varphi_{3}\right)=-\bar{h}_{23} \varphi_{3}+s_{32}, \\
& V_{1}\left(\varphi_{1}\right)=v_{1}-\left(\bar{s}_{11}+r_{21}+\bar{s}_{21}+r_{31}+\bar{s}_{31}\right) \varphi_{1} \\
& V_{2}\left(\varphi_{2}\right)=v_{2}-\left(\bar{s}_{22}+r_{12}+\bar{s}_{12}+r_{32}+\bar{s}_{32}\right) \varphi_{2} \\
& V_{3}\left(\varphi_{3}\right)=-\bar{v}_{1}-\bar{v}_{2}-\left(\bar{s}_{33}+r_{13}+\bar{s}_{13}+r_{23}+\bar{s}_{23}\right) \varphi_{3},
\end{aligned}
$$

where $v_{i}, h_{i j}, g_{i j}, r_{i j}$, and $s_{i j}$ are complex constants in general except that $h_{11}, h_{22}$ and $h_{33}$ are real. Another lemma which will be useful in the derivation to follow is also given here.

Lemma 2. Let $F_{k}\left(\boldsymbol{x}, \boldsymbol{u}, \varphi_{k}\right)$ be given by

$$
\left.F_{k}=2 \mathrm{i} p_{k 2}\left(\sum_{i=1}^{3} A_{i k} R_{, u_{i}}\right) \varphi_{k}+\delta_{1 k}\left(R_{, 2}-\bar{p}_{1} R_{, 1}\right) \quad \text { (no sum over } k\right),
$$

where $R(\boldsymbol{x}, \boldsymbol{u})$ is an arbitrary smooth function of $\boldsymbol{x}$ and $\boldsymbol{u}, A$ is given by (2.7) and $\delta_{1 k}$ is the Kronecker delta. The corresponding densities $T_{1}$ and $T_{2}$ form the components of a trivial conservation law.

It is not difficult to verify Lemma 2 by substituting the expression for $\varphi_{k}$ in (2.22) and (2.23) and the orthogonal conditions (2.15) and (2.16) into (3.25).

Now by expanding (3.24) and using Lemma 1 , we have the following equations:

$$
\begin{aligned}
& 2 \mathrm{i}\left(p_{1} / p_{12}\right) F_{1, z_{1}}=v_{1}-\left(\bar{s}_{11}+r_{21}+\bar{s}_{21}+r_{31}+\bar{s}_{31}\right) \varphi_{1}, \\
& \left(\mathrm{i} / p_{12}\right)\left(p_{1}-\bar{p}_{1}\right)\left(\bar{A}_{11} F_{1, u_{1}}+\bar{A}_{21} F_{1, u_{2}}+\bar{A}_{31} F_{1, u_{3}}\right)=\mathrm{i} h_{11} \varphi_{1}+s_{11}, \\
& \left(\mathrm{i} / p_{12}\right)\left(p_{1}-p_{2}\right)\left(A_{12} F_{1, u_{1}}+A_{22} F_{1, u_{2}}+A_{32} F_{1, u_{3}}\right)=g_{12} \varphi_{1}+r_{12}, \\
& \left(\mathrm{i} / p_{12}\right)\left(p_{1}-\bar{p}_{2}\right)\left(\bar{A}_{12} F_{1, u_{1}}+\bar{A}_{22} F_{1, u_{2}}+\bar{A}_{32} F_{1, u_{3}}\right)=h_{12} \varphi_{1}+s_{12}, \\
& \left(\mathrm{i} / p_{12}\right)\left(p_{1}-p_{3}\right)\left(A_{13} F_{1, u_{1}}+A_{23} F_{1, u_{2}}+A_{33} F_{1, u_{3}}\right)=g_{13} \varphi_{1}+r_{13}, \\
& \left(\mathrm{i} / p_{12}\right)\left(p_{1}-\bar{p}_{3}\right)\left(\bar{A}_{13} F_{1, u_{1}}+\bar{A}_{23} F_{1, u_{2}}+\bar{A}_{33} F_{1, u_{3}}\right)=h_{13} \varphi_{1}+s_{13},
\end{aligned}
$$

Proc. R. Soc. Lond. A (1993) 
$2 \mathrm{i}\left(p_{2} / p_{22}\right) F_{2, \tilde{z}_{2}}=v_{2}-\left(\bar{s}_{12}+r_{12}+\bar{s}_{22}+r_{32}+\bar{s}_{32}\right) \varphi_{2}$,

8

$\left(\mathrm{i} / p_{22}\right)\left(p_{2}-\bar{p}_{2}\right)\left(\bar{A}_{12} F_{2, u_{1}}+\bar{A}_{22} F_{2, u_{2}}+\bar{A}_{32} F_{2, u_{3}}\right)=\mathrm{i} h_{22} \varphi_{2}+s_{22}$,

9

10

$\left(\mathrm{i} / p_{22}\right)\left(p_{2}-p_{1}\right)\left(A_{11} F_{2, u_{1}}+A_{21} F_{2, u_{2}}+A_{31} F_{2, u_{3}}\right)=-g_{12} \varphi_{2}+r_{21}$,

11

$\left(\mathrm{i} / p_{22}\right)\left(p_{2}-\bar{p}_{1}\right)\left(\bar{A}_{11} F_{2, u_{1}}+\bar{A}_{21} F_{2, u_{2}}+\bar{A}_{31} F_{2, u_{3}}\right)=-\bar{h}_{12} \varphi_{2}+s_{21}$,

12

$\left(\mathrm{i} / p_{22}\right)\left(p_{2}-p_{3}\right)\left(A_{13} F_{2, u_{1}}+A_{23} F_{2, u_{2}}+A_{33} F_{2, u_{3}}\right)=g_{23} \varphi_{2}+r_{23}$,

13

$\left(\mathrm{i} / p_{22}\right)\left(p_{2}-\bar{p}_{3}\right)\left(\bar{A}_{13} F_{2, u_{1}}+\bar{A}_{23} F_{2, u_{2}}+\bar{A}_{33} F_{2, u_{3}}\right)=h_{23} \varphi_{2}+s_{23}$,

14

15

16

17

18 $2 \mathrm{i}\left(p_{3} / p_{32}\right) F_{3, \tilde{z}_{3}}=-\bar{v}_{1}-\bar{v}_{2}-\left(\bar{s}_{33}+r_{13}+\bar{s}_{13}+r_{23}+\bar{s}_{23}\right) \varphi_{3}$,

First, consider 1, 7 and 13, we can set

$$
\left.\begin{array}{l}
F_{1}=F_{1}^{*}\left(z_{1}, \varphi_{1}\right)+w_{1}(\boldsymbol{x}, \boldsymbol{u}) \varphi_{1}+w_{10}(\boldsymbol{x}, \boldsymbol{u}), \\
F_{2}=F_{2}^{*}\left(z_{2}, \varphi_{2}\right)+w_{2}(\boldsymbol{x}, \boldsymbol{u}) \varphi_{2}+w_{20}(\boldsymbol{x}, \boldsymbol{u}), \\
F_{3}=F_{3}^{*}\left(z_{3}, \varphi_{3}\right)+w_{3}(\boldsymbol{x}, \boldsymbol{u}) \varphi_{3}+w_{30}(\boldsymbol{x}, \boldsymbol{u}),
\end{array}\right\}
$$

where $w_{k}$ and $w_{k 0}, k=1-3$, are arbitrary functions. Next, since $h_{11}, h_{22}$ and $h_{33}$ are real functions, then from 2,8 and 14 , we can further set $w_{1}, w_{2}$ and $w_{3}$ as follows:

$$
\left.\begin{array}{l}
w_{1}=2 \mathrm{i} p_{12}\left(A_{11} R_{1, u_{1}}+A_{21} R_{1, u_{2}}+A_{31} R_{1, u_{3}}\right), \\
w_{2}=2 \mathrm{i} p_{22}\left(A_{12} R_{2, u_{1}}+A_{22} R_{2, u_{2}}+A_{32} R_{2, u_{3}}\right), \\
w_{3}=2 \mathrm{i} p_{32}\left(A_{13} R_{3, u_{1}}+A_{23} R_{3, u_{2}}+A_{33} R_{3, u_{3}}\right),
\end{array}\right\}
$$

where $R_{1}(\boldsymbol{x}, \boldsymbol{u}), R_{2}(\boldsymbol{x}, \boldsymbol{u})$ and $R_{3}(\boldsymbol{x}, \boldsymbol{u})$ are arbitrary smooth real functions. Further investigations of 3 and 9,4 and 10,5 and 15 , etc., allow us to set $R_{1}=$ $R_{2}=R_{3}=R(\boldsymbol{x}, \boldsymbol{u})$ except for the terms that depend only on $\boldsymbol{x}$. But the contributions of $w_{i}$ to $F_{i}$ result in trivial conservation laws if we compare (3.27) with Lemma 2. Thus the forms of $F_{1}, F_{2}$ and $F_{3}$ for non-trivial conservation laws can be expressed as

$$
\left.\begin{array}{l}
F_{1}=F_{1}^{*}\left(z_{1}, \varphi_{1}\right)+w_{1}^{*}(\boldsymbol{x}) \varphi_{1}+w_{10}(\boldsymbol{x}, \boldsymbol{u}), \\
F_{2}=F_{2}^{*}\left(z_{2}, \varphi_{2}\right)+w_{2}^{*}(\boldsymbol{x}) \varphi_{2}+w_{20}(\boldsymbol{x}, \boldsymbol{u}), \\
F_{3}=F_{3}^{*}\left(z_{3}, \varphi_{3}\right)+w_{3}^{*}(\boldsymbol{x}) \varphi_{3}+w_{30}(\boldsymbol{x}, \boldsymbol{u}) .
\end{array}\right\}
$$

It is not difficult to deduce that $w_{10}, w_{20}$ and $w_{30}$ are at most linear in $\boldsymbol{u}$ by first comparing the coefficients of $\varphi_{1}, \varphi_{2}$ and $\varphi_{3}$ in $1,7,13$, respectively, and, next, the other remaining equations. According to Olver (1988), the type of conserved density $\boldsymbol{T}$ which is linear in $\boldsymbol{u}$ and $\nabla \boldsymbol{u}$ must be equivalent to, except for trivial densities, Betti's reciprocal law whose components are given by

$$
T_{1}=\sigma_{i 1} u_{i}^{*}-\sigma_{i 1}^{*} u_{i}, \quad T_{2}=\sigma_{i 2} u_{i}^{*}-\sigma_{i 2}^{*} u_{i}
$$

in which

$$
\sigma_{i j}=C_{i j k l} u_{k, l}, \quad \sigma_{i j}^{*}=C_{i j k l} u_{k, l}^{*}
$$

$\boldsymbol{u}^{*}(\boldsymbol{x})$ is any solution of (2.1). In summary the general first-order conservation Proc. R. Soc. Lond. A (1993) 
laws for non-degenerate anisotropic materials with components $T_{1}(\boldsymbol{x}, \boldsymbol{u}, \nabla \boldsymbol{u})$ and $T_{2}(\boldsymbol{x}, \boldsymbol{u}, \nabla \boldsymbol{u})$ are of the following form:

$$
\begin{aligned}
T_{1}(\boldsymbol{x}, \boldsymbol{u}, \nabla \boldsymbol{u}) & =T_{1}^{o}+T_{1}^{*}+\sum_{k=1}^{3}\left(F_{k 1}+\frac{p_{k 1}}{p_{k 2}} F_{k 2}\right), \\
T_{2}(\boldsymbol{x}, \boldsymbol{u}, \nabla \boldsymbol{u}) & =T_{2}^{o}+T_{2}^{*}+\sum_{k=1}^{3} \frac{-1}{p_{k 2}} F_{k 2}, \\
F_{k}\left(z_{k}, \varphi_{k}\right) & =F_{k 1}+\mathrm{i} F_{k 2},
\end{aligned}
$$

where $T_{1}^{o}$ and $T_{2}^{o}$ are the components of trivial conservation laws; $T_{1}^{*}$ and $T_{2}^{*}$ are the components of Betti's law given in (3.29) and (3.30); $F_{k}\left(z_{k}, \varphi_{k}\right)$ are arbitrary analytic functions of their corresponding complex arguments.

Thus, except for Betti's law, there are three infinite families of conservation laws, each depending on an arbitrary analytic function $F_{k}\left(z_{k}, \varphi_{k}\right)$ of two complex variables if Stroh's eigenvalues $p_{\alpha}$ are distinct. For degenerate materials, the conservation laws can still be constructed by modifying the form of the general solutions of the system. For simplicity, we only consider the case where $p$ is a double root here. The higher order multiple roots can be treated in a similar way without difficulty. If $p$ is a double root, the second independent solution of (2.1) can be set as follows (cf. Ting 1982):

$$
\begin{gathered}
u_{i}=a_{i} \tilde{z} \hat{f}_{2}^{\prime}(z)+a_{i}^{*} \hat{f}_{2}(z), \\
z=x_{1}+p x_{2}, \quad \tilde{z}=x_{1}-p x_{2},
\end{gathered}
$$

where it can be shown directly that $\boldsymbol{a}$ is the eigenvector in (2.3) associated with the double root $p$ and $\hat{f}_{2}(z)$ is an arbitrary analytic function with complex argument $z$. The vector $\boldsymbol{a}^{*}$ can be determined by substituting into (2.1)

$$
\left(\boldsymbol{Q}+p\left(\boldsymbol{R}+\boldsymbol{R}^{\mathrm{T}}\right)+p^{2} \boldsymbol{T}\right) \boldsymbol{a}^{*}=-2\left(\boldsymbol{Q}-p^{2} \boldsymbol{T}\right) \boldsymbol{a},
$$

where the matrices $\boldsymbol{Q}, \boldsymbol{R}$ and $\boldsymbol{T}$ are given by (2.4). However, the determinant of the coefficient matrix of the vector $\boldsymbol{a}^{*}$ is zero for this double root $p$, the existence of the vector $\boldsymbol{a}^{*}$ can hold if and only if

$$
\boldsymbol{a}^{\mathrm{T}}\left(\boldsymbol{Q}-p^{2} \boldsymbol{T}\right) \boldsymbol{a}=0 .
$$

Further, it follows from (2.3) that the criterion given by (3.37) can be replaced by

$$
\boldsymbol{a}^{\mathrm{T}}\left\{\left(\boldsymbol{R}+\boldsymbol{R}^{\mathrm{T}}\right)+2 p \boldsymbol{T}\right\} \boldsymbol{a}=0
$$

for the existence of the vector $\boldsymbol{a}^{*}$.

To illustrate the procedure for degenerate materials, we consider the case of linear homogeneous isotropic plane elasticity with the governing equations

$$
\left.\begin{array}{l}
(\lambda+2 \mu) u_{1,11}+\mu u_{1,22}+(\lambda+\mu) u_{2,12}=0, \\
(\lambda+\mu) u_{1,12}+\mu u_{2,11}+(\lambda+2 \mu) u_{2,22}=0,
\end{array}\right\}
$$

where $\lambda, \mu$ are Lamé constants. The first independent solution of (3.39) can be Proc. R. Soc. Lond. A (1993) 
obtained easily as

$$
\begin{gathered}
u_{i}=a_{i} \hat{f}_{1}(z), z=x_{1}+\mathrm{i} x_{2}, \\
\boldsymbol{a}=-\left\{\begin{array}{c}
1 \\
\mathrm{i}
\end{array}\right\} .
\end{gathered}
$$

However, the root $p=\mathrm{i}$ are repeated. It can be shown that the eigenvector $\boldsymbol{a}$ in (3.41) satisfies the criterion of (3.38) so that the existence of the second independent solution is ensured. The vector $\boldsymbol{a}^{*}$ in (3.36) can be chosen as

$$
\boldsymbol{a}^{*}=(3-4 \nu)\left\{\begin{array}{c}
1 \\
-i
\end{array}\right\}
$$

where $\nu$ is Poisson's ratio related to $\lambda$ and $\mu$ by $\lambda=2 \mu \nu /(1-2 \nu)$. The general solution of linear homogeneous isotropic plane elasticity can thus be expressed as follows:

$$
2 \mu\left\{\begin{array}{l}
u_{1} \\
u_{2}
\end{array}\right\}=\operatorname{Re}\left[\left\{\begin{array}{l}
-1 \\
-\mathrm{i}
\end{array}\right\} \hat{f}_{1}(z)+\left\{\begin{array}{l}
-1 \\
-\mathrm{i}
\end{array}\right\} \bar{z} \hat{f}_{2}^{\prime}(z)+(3-4 \nu)\left\{\begin{array}{c}
1 \\
-\mathrm{i}
\end{array}\right\} \hat{f}_{2}(z)\right],
$$

where $\hat{f}_{1}(z)$ and $\hat{f}_{2}(z)$ are arbitrary analytic function with argument $z=x_{1}+\mathrm{i} x_{2}$; moreover, this form of solutions given by (3.43) is identical with the famous one proposed by Muskhelishvili (1953).

The first order conservation laws of linear isotropic plane elasticity can be developed in a similar way treated in the first part of this section except that the general solution given by (2.9) is replaced by (3.43). In fact, all the first-order conservation laws of the system of (3.39) have been derived by Olver (1984) and we list the primary results here with minor modifications. Differentiating (3.43) with respect to the coordinates $x_{1}$ and $x_{2}$, respectively, gives

$$
\begin{aligned}
& \hat{\xi}=\bar{z} \hat{f}_{2}^{\prime \prime}(z)+\hat{f}_{1}^{\prime}(z)=\mu\left\{\left(u_{2,2}-u_{1,1}\right)+\mathrm{i}\left(u_{1,2}+u_{2,1}\right)\right\}, \\
& \hat{\eta}=\hat{f}_{2}^{\prime}(z)=\{4(1-\nu)\}^{1 / 2}\left\{(\lambda+2 \mu)\left(u_{1,1}+u_{2,2}\right)+\mathrm{i} \mu\left(u_{2,1}-u_{1,2}\right)\right\} .
\end{aligned}
$$

Then, according to the similar arguments for the fully anisotropic case, we can change variables from $u_{1,1}, u_{1,2}, u_{2,1}$ and $u_{2,2}$ to the real and imaginary parts of $\hat{\xi}$ and $\hat{\eta}$ respectively. The fact that $\hat{\eta}$ is an analytic function of $z$ and the relation $\partial \hat{\xi} / \partial \bar{z}=\hat{\eta}^{\prime}$ from (3.44) and (3.45) results in

$$
\begin{gathered}
\hat{\eta}_{1,1}=\hat{\eta}_{2,2}, \quad \hat{\eta}_{1,2}=-\hat{\eta}_{2,1}, \\
{[2 p t] \hat{\xi}_{1,1}-\hat{\xi}_{2,2}=2 \hat{\eta}_{1,1}} \\
\hat{\xi}_{1,2}+\hat{\xi}_{2,1}=2 \hat{\eta}_{2,1} .
\end{gathered}
$$

Moreover, let the components of the conserved density $\boldsymbol{T}$ be $T_{1}$ and $T_{2}$ respectively. $\boldsymbol{x}-\boldsymbol{u}$ independent conservation laws of the system (3.39) can be obtained by first substituting the general solution (3.43) and the relations (3.46)-(3.46) into the expansion of (3.1) treating $\hat{\xi}_{1,1}, \hat{\xi}_{2,1}, \hat{\eta}_{1,1}$ and $\hat{\eta}_{2,1}$ as independent variables. From this approach and without repeating the similar derivations proposed by Olver in the subsequent proofs, all the first-order conservation laws depending on the material coordinates and deformation gradients are given by (Olver 1986)

$$
F=-\hat{\xi} \frac{\partial G}{\partial \hat{\eta}}+\left(\bar{G}-\bar{z} \frac{\partial G}{\partial z}\right)+H
$$

Proc. R. Soc. Lond. A (1993) 
where

$$
F=T_{1}-\mathrm{i} T_{2}, \quad G=G(z ; \hat{\eta}), \quad H=H(z ; \hat{\eta})
$$

In addition, except for Betti's reciprocal law, the extra conservation law not present in the anisotropic case forms the well-known $L$-integral and is given by (cf. Olver 1984b)

$$
F=\mathrm{i}\left\{[(1-\nu) / \mu]\left(\bar{z} \hat{\eta}^{2}-2 z \hat{\xi} \hat{\eta}-\bar{z} \overline{\hat{\eta}}^{2}\right)+w \hat{\xi}+(\hat{\eta}+\overline{\hat{\eta}}) \bar{w}\right\}
$$

where $w=u_{1}+\mathrm{i} u_{2}$.

\section{Explicit real forms}

With the forms of $T_{1}$ and $T_{2}$ given by (3.31) and (3.32), respectively, any integral form of conservation law which depends on the coordinates and the first derivatives of dependent variables can be written in terms of the associated generating complex functions

$$
\int\left(T_{1} \mathrm{~d} x_{2}-T_{2} \mathrm{~d} x_{1}\right)=\operatorname{Im}\left(\sum_{k} \frac{1}{p_{k 2}} \int F_{k} \mathrm{~d} z_{k}\right)=0,
$$

where Im denotes the imaginary part. Equation (4.1) is valid for arbitrary closed integration contour enclosing a region where $\nabla \cdot \boldsymbol{T}=0$. Moreover, by letting $F_{k}=p_{k 2} \tilde{F}_{k}$ or $F_{k}=\mathrm{i} p_{k 2} \tilde{F}_{k}$, it is readily seen that

$$
\int \sum_{k} \tilde{F}_{k} \mathrm{~d} z_{k},=0
$$

This is consistent with the fact that $\tilde{F}_{k}$ is an analytic function of $z_{k}$ and integration of an analytic function over a closed contour around a region of analyticity of $\tilde{F}_{k}$ vanishes. The analysis here thus shows that the conservation laws are equivalent to the well-known Cauchy's integral theorem for analytic functions.

The complex form of the conservation laws can be conveniently separated into two real integrals in terms of the physical quantities such as displacements and tractions by utilizing the expression for $\varphi_{k}$ given by (2.22) and the orthogonality relations given by $(2.13)-(2.16)$. For example, letting $\tilde{F}_{k}=\varphi_{k}^{2}$ or $\tilde{F}_{k}=z_{k} \varphi_{k}^{2}$, respectively, leads to

$$
\begin{gathered}
J-\mathrm{i} \hat{J}=\sum_{k} \int \varphi_{k}^{2} \mathrm{~d} z_{k}, \\
M-\mathrm{i} \hat{M}=\sum_{k} \int z_{k} \varphi_{k}^{2} \mathrm{~d} z_{k} .
\end{gathered}
$$

Equations (4.2) and (4.3) can be expressed in the following forms:

$$
\begin{gathered}
J-\mathrm{i} \hat{J}=\sum_{k} \int \frac{\partial f_{k}}{\partial x_{1}} \frac{\partial f_{k}}{\partial s} \mathrm{~d} s, \\
M-\mathrm{i} \hat{M}=\sum_{k} \int r \frac{\partial f_{k}}{\partial r} \frac{\partial f_{k}}{\partial s} \mathrm{~d} s,
\end{gathered}
$$

where $s$ is the arc length along the integration contour, $r=\sqrt{ }\left(x_{1}^{2}+x_{2}^{2}\right)$ and Proc. R. Soc. Lond. A (1993) 
$f_{k}$ are Stroh's functions. Use of the expression for $f_{k}$ given by $(2.20)$ and the orthogonality relations given by $(2.13)-(2.16)$ then gives

$$
\begin{aligned}
\hat{J} & =\frac{1}{2} \int\left\{\left(\psi_{i, 1} H_{i j} \frac{\partial \psi_{j}}{\partial s}\right)+\left(\frac{\partial \psi_{i}}{\partial s} S_{i j} u_{j, 1}+\psi_{i, 1} S_{i j} \frac{\partial u_{j}}{\partial s}\right)-\left(\frac{\partial u_{i}}{\partial s} L_{i j} u_{j, 1}\right)\right\} \mathrm{d} s \\
J & =\frac{1}{2} \int\left(u_{i, 1} \frac{\partial \psi_{i}}{\partial s}+\psi_{i, 1} \frac{\partial u_{i}}{\partial s}\right) \mathrm{d} s \\
\hat{M} & =\frac{1}{2} \int\left\{\left(\frac{\partial \psi_{i}}{\partial r} H_{i j} \frac{\partial \psi_{j}}{\partial s}\right)+\left(\frac{\partial \psi_{i}}{\partial s} S_{i j} \frac{\partial u_{j}}{\partial r}+\frac{\partial \psi_{i}}{\partial r} S_{i j} \frac{\partial u_{j}}{\partial s}\right)-\left(\frac{\partial u_{i}}{\partial s} L_{i j} \frac{\partial u_{j}}{\partial r}\right)\right\} r \mathrm{~d} s \\
M & =\frac{1}{2} \int\left(\frac{\partial u_{i}}{\partial r} \frac{\partial \psi_{i}}{\partial s}+\frac{\partial \psi_{i}}{\partial r} \frac{\partial u_{i}}{\partial s}\right) r \mathrm{~d} s
\end{aligned}
$$

in which the matrices $\boldsymbol{H}, \boldsymbol{L}$ and $\boldsymbol{S}$ are given by (2.17), (2.18) and (2.19) respectively. The $J$ and $M$ integrals are the well-known conservation integrals derived by Knowels \& Sternberg (1972) in alternative forms. The $\hat{J}$ and $\hat{M}$ integrals are new. However, it is unclear at this point what physical significance can be attached to these new integrals. Note that since real-form integrals shown above are expressed directly in terms of quantities that do not depend on Stroh's eigenvalue $p$, they are also valid for degenerate materials such as isotropic materials.

Equations (4.2) and (4.3) can be generalized to obtain interactive conservation laws involving two elastic states as

$$
\begin{gathered}
J_{\text {int }}-\mathrm{i} \hat{J}_{\text {int }}=\sum_{k} \int \frac{\partial f_{k}^{*}}{\partial x_{1}} \frac{\partial f_{k}}{\partial s} \mathrm{~d} s \\
M_{\text {int }}-\mathrm{i} \hat{M}_{\text {int }}=\sum_{k} \int r \frac{\partial f_{k}^{*}}{\partial r} \frac{\partial f_{k}}{\partial s} \mathrm{~d} s
\end{gathered}
$$

where $f$ and $f^{*}$ are Stroh's functions for two arbitrary elastic states. The corresponding real-form expressions are given by

$$
\begin{aligned}
\hat{J}_{\mathrm{int}}= & \frac{1}{2} \int\left\{\left(\psi_{i, 1}^{*} H_{i j} \frac{\partial \psi_{j}}{\partial s}\right)+\left(\frac{\partial \psi_{i}}{\partial s} S_{i j} u_{j, 1}^{*}+\psi_{i, 1}^{*} S_{i j} \frac{\partial u_{j}}{\partial s}\right)-\left(\frac{\partial u_{i}}{\partial s} L_{i j} u_{j, 1}^{*}\right)\right\} \mathrm{d} s, \\
J_{\mathrm{int}}= & \frac{1}{2} \int\left(u_{i, 1}^{*} \frac{\partial \psi_{i}}{\partial s}+\psi_{i, 1}^{*} \frac{\partial u_{i}}{\partial s}\right) \mathrm{d} s \\
\hat{M}_{\mathrm{int}}= & \frac{1}{2} \int\left\{\left(\frac{\partial \psi_{i}^{*}}{\partial r} H_{i j} \frac{\partial \psi_{j}}{\partial s}\right)+\left(\frac{\partial \psi_{i}}{\partial s} S_{i j} \frac{\partial u_{j}^{*}}{\partial r}+\frac{\partial \psi_{i}^{*}}{\partial r} S_{i j} \frac{\partial u_{j}}{\partial s}\right)\right. \\
& \left.\quad-\left(\frac{\partial u_{i}}{\partial s} L_{i j} \frac{\partial u_{j}^{*}}{\partial r}\right)\right\} r \mathrm{~d} s, \\
M_{\mathrm{int}}= & \frac{1}{2} \int\left(\frac{\partial u_{i}^{*}}{\partial r} \frac{\partial \psi_{i}}{\partial s}+\frac{\partial \psi_{i}^{*}}{\partial r} \frac{\partial u_{i}}{\partial s}\right) r \mathrm{~d} s
\end{aligned}
$$

Similar two-state conservation laws have been discussed in Chen \& Shield (1977) and Gurtin (1977). 


\section{Concluding remarks}

In this paper we have presented a complete class of first-order conservation laws in general anisotropic elasticity. The class of conservation laws are valid for deformations in which three displacement components are present but are dependent only on two coordinates. Based on Stroh's formalism for anisotropic elasticity, we have shown that the conservation laws are closely related to Cauchy's theorem for complex analytic functions. The orthogonality relations in Stroh's formalism were utilized to transform the complex conservation laws into those in real forms. The real-form integrals contain only quantities that do not depend on Stroh's eigenvalues and hence are valid for either non-degenerate or degenerate materials. In an accompanying paper we shall extend the present derivations to include thermoelasticity with some application of the conservation laws to crack problems.

\section{References}

Barnett, D. M. \& Lothe, J. 1973 Synthesis of the sextic and the integral formalism for dislocation, Green's function and surface waves in anisotropic elastic solids. Phys. Norv. 7, 13-19.

Chadwick, P. \& Smith, G. D. 1977 Foundations of the theory of surface waves in anisotropic elastic materials. Adv. appl. Mech. 17, 303-376.

Chen, F. H. K. \& Shield, R. T. 1977 Conservation laws in elasticity of the J-integral type. ZAMP J. appl. Math. Phys. 28, 1-22.

Eshelby, J. D. 1956 The continuum theory of lattice defects. In Solid state physics (ed. F. Seitz \& D. Turnbull), vol. III. New York: Academic Press.

Gurtin, M. E. 1977 Some conservation laws in linear elasticity for pairs of fields. Int. J. Fracture 13, 391-393.

Knowles, J. K. \& Sternberg, E. 1972 On a class of conservation laws in linearized and finite elastostatics. Arch. ration. Mech. Analysis 44, 187-211.

Muskhelishvili, N. I. 1953 Some basic problems of the mathematical theory of elasticity. Groningen, The Netherlands: Noordhoff.

Noether, E. 1918 Invariante Variationsprobleme. Nachr. Ges. Göttingen, Math.-Phys. Kl. 2, 235-257.

Olver, P. J. 1984 a Conservation laws in elasticity. I. General results. Arch. ration. Mech. Analysis 85, 119-129.

Olver, P. J. $1984 b$ Conservation laws in elasticity. II. Linear homogeneous isotropic elastostatics. Arch. ration. Mech. Analysis 85, 131-160.

Olver, P. J. 1986 Applications of Lie groups to differential equations. Graduate texts in mathematics, vol. 107. New York: Springer-Verlag.

Olver, P. J. 1988 Conservation laws in elasticity. III. Planar linear anisotropic elastostatics. Arch. ration. Mech. Analysis 102, 167-181.

Rice, J. R. 1968 A Path-independent integral and the approximate analysis of strain concentration by notches and cracks. ASME J. appl. Mech. 35, 379-386.

Stroh, A. N. 1958 Dislocations and cracks in anisotropic elasticity. Phil. Mag. 3, 625-646.

Stroh, A. N. 1962 Steady-state problems in anisotropic elasticity. J. Math. Phys. 41, 77-103.

Ting, T. C. T. 1982 Effects of changes of references coordinates on the stress analyses of anisotropic elastic materials. Int. J. Solids Struct. 18, 139-152.

Ting, T. C. T. 1986 Explicit solution and invariance of the singularities at the interface crack in anisotropic composites. Int. J. Solids Struct. 9, 965-983. 